\title{
Serotonin transporter polymorphisms and clinical response to sertraline across ethnicities
}

\begin{abstract}
The aim of this pilot study was to examine the relationship between clinical response, adverse effects, sertraline (SERT) plasma concentrations and the genetic polymorphism of the serotonin transporter gene-linked polymorphic region (5HTTLPR) in 2 ethnic patient groups. The study involved 45 patients in a clinical trial who received a fixed dose regimen of $50 \mathrm{mg}$ SERT for one week, then a variable-dose regimen for a further 6 weeks for major depressive disorder. At weeks 1 and 6, the following assessments were completed: Hamilton Depression Rating Scale (HDRS), Clinical Global Impression (CGI), drug adverse reaction scale and measurement of plasma SERT levels. Genomic analysis for the long and short allele variants of the 5HTTLPR polymorphism was also carried out. Caucasian subjects had a higher rate of 1/1 genotype while Chinese subjects had higher frequencies of $1 / \mathrm{s}$ and $\mathrm{s} / \mathrm{s}$ genotypes. Comparison of the subjects with the 5HTTLPR s/s genotype and those with the $1 / 1$ and $1 / \mathrm{s}$ genotypes found no significant differences in the HDRS scores, CGI scores, response rates, adverse effects and SERT plasma concentrations at week 6.
\end{abstract}

Keyword: Adverse effects; 5-HTT polymorphisms; Plasma concentrations; Sertraline 